\title{
PHYSICAL OPTICS
}

\section{On Diffraction of Radiation Packets with Ultimately Short Duration}

\author{
N. N. Rosanov ${ }^{a, b}$ and G. B. Sochilin ${ }^{a}$ \\ ${ }^{a}$ Vavilov State Optical Institute, St. Petersburg, 199034 Russia \\ ${ }^{b}$ National Research University of Information Technologies, Mechanics and Optics, St. Petersburg, 197101 Russia \\ e-mail: nrosanov@yahoo.com \\ Received February 03, 2012
}

\begin{abstract}
The inference of the work by V.A. Banakh that there is an absence of diffraction of optical radiation in the limit of zero pulse duration is analyzed.
\end{abstract}

DOI: $10.1134 / \mathrm{S} 0030400 \mathrm{X} 12070181$

In recent work [1], the diffraction of an optical radiation packet was theoretically analyzed in the limit of tending to zero of its duration (the time dependence of the field amplitude in the form of the Dirac $\delta$-function is implied). The principal conclusion of [1] is that diffraction of focused radiation vanishes in this limit of the " $\delta$-pulse."

The abovementioned conclusion of [1] was obtained with the use of not only the quasi-optical approximation, but also in terms of the complete scalar wave equation. Although the calculations given in [1] give rise to objections, it is more significant that the ultimate conclusion is obvious, and even trivial, for the model considered. Indeed, let us retrace the limiting transition to a pulse of a "zero" duration, $T \rightarrow 0$. Initially in [1], the Gaussian time profile of the field amplitude for the pulse of duration $T$ is specified,

$$
|G(t)|=G_{0} \exp \left(-\frac{t^{2}}{2 T^{2}}\right)
$$

Here, $t$ is the time and $G_{0}$ is the maximal amplitude. In order for the pulse energy to remain constant in the limit $T \rightarrow 0$, it is necessary that

$$
G_{0} \sim T^{-1 / 2} \text {. }
$$

As is known, for monochromatic radiation, the diffraction length of a beam is directly proportional to the radiation frequency [2]. The width of the radiation spectrum of the pulse is $\Delta \omega \sim T^{-1}$; therefore, the spectrum is unrestrictedly wide in the limit $T \rightarrow 0$ and almost all of the energy is contained in radiation with infinitely high frequencies. Clearly, the diffraction is indeed absent for these frequencies (the diffraction length becomes infinite). It should be also noted that the introduction of the carrier frequency $\omega_{0}$ in this limit loses its meaning.

Thus, the inference of [1] that there is an absence of diffraction of the $\delta$-pulse is equivalent to the wellknown statement that there is an increase of the diffraction length with increasing radiation frequency [2]. The $\delta$-pulse model itself in the form used in [1] seems to be nonphysical and unfit for the description of diffraction of ultimately short pulse radiation.

\section{REFERENCES}

1. V. A. Banakh, Opt. Lett. 36 (23), 4539 (2011).

2. M. B. Vinogradova, O. V. Rudenko, and A. P. Sukhorukov, Theory of Waves (Nauka, Moscow, 1979). 